A. Bayo Salas, J. Beerten, D. Van Hertem, "Analytical methodology to develop frequencydependent equivalents in networks with multiple converters," in Proc. IEEE PowerTech 2017, Manchester, UK, Jun. 18-22, 2017, 7 pages.

Digital Object Identifier: $\underline{\text { 10.1109/PTC.2017.7980938 }}$

URL:

https://ieeexplore.ieee.org/document/7980938

(C) 2017 IEEE. Personal use of this material is permitted. Permission from IEEE must be obtained for all other users, including reprinting/ republishing this material for advertising or promotional purposes, creating new collective works for resale or redistribution to servers or lists, or reuse of any copyrighted components of this work in other works. 


\section{Analytical methodology to develop frequency-dependent equivalents in networks with multiple converters}

\author{
Alejandro Bayo-Salas \\ $R \& D$ department \\ Elia System Operator \\ Brussels, Belgium \\ Alejandro.BayoSalas@elia.be
}

\author{
Jef Beerten \\ Dirk Van Hertem \\ ELECTA \\ EnergyVille-University of Leuven (KU Leuven) \\ Leuven, Belgium
}

\begin{abstract}
This paper discusses the analysis of dynamic interactions above the fundamental frequency in networks with high penetration of converters. For this purpose, interaction studies require a good representation of network resonances and converter dynamics. However, current methodologies present some limitations in this respect. On one hand, the harmonic impedance obtained from the frequency scan in software does not consider the influence of converter control dynamics. On the other hand, typical stability studies consider those at the cost of simplifying the network representation. The increasing penetration of converters in the network arises concerns due to their limitation in accurately capturing the instability. This paper demonstrates the impact of control interactions in a meshed network with several converters and how this interaction influences the network resonances. Due to the limitations of existing methods, the paper presents a systematic method to capture the relevant dynamic phenomena.
\end{abstract}

Index Terms-VSC-HVDC, System stability, Harmonic interactions, EMT analysis.

\section{INTRODUCTION}

The increasing integration of new types of generation coupled to the network through power electronics and the installation of High-Voltage Direct Current (HVDC) links based on voltage source converters (VSC) are driving the most important change in the power system since it was built These new technologies based on power electronics offer the flexibility to rapidly control power flows and support the AC system.

This controllability leads to an extension of the frequency range of the dynamic control loops. This range is wider than the control bandwidth in conventional installations, usually located below the fundamental frequency. Therefore, VSC control dynamics start interacting with electro-magnetic dynamics from the network.

Alejandro Bayo-Salas is funded by the People Programme (Marie Curie Actions) of the EU's Seventh Framework Programme FP7/2007-2013/ under REA grant agreement no. 317221, project title MEDOW. Jef Beerten is funded by a post-doctoral fellowship from the Research Foundation Flanders (FWOVlaanderen)
Similar interactions have already led to stability problems in traction networks [1], [2] and in distribution systems [3], [4]. Also in the high-voltage installations, first worldwide installations of multi-modular converters (MMC)-HVDC have experienced these problems in a HVDC-connected offshore wind farm [5] and a point-to-point DC link in continental Europe [6]. In all the reported cases, instabilities appeared due to an interaction between the control and a system resonance.

For this reason, utilities are typically requested to provide the manufacturer with the harmonic impedance of the network as seen from the point of connection [7], [8]. This harmonic impedance is usually obtained from commercial power system analysis programs by applying a frequency sweep to the network at the point of connection of the new equipment. However, this obtained impedance only characterizes the frequency-dependent (FD) behaviour of the represented passive network components. This implies that, in case other controlled devices are present in the network, their control dynamics would be neglected and, thus, the network frequencydependent behaviour might not be accurately represented.

Recent literature shows that the influence of control dynamics of parallel-connected converters in the overall dynamic response of the network. Examples include studies in offshore wind farms [9]-[12], between two converters connected through a tie-line [13], [14], and between three converters in a simple meshed grid [15]. In these publications, the network has typically been represented by simplified models such as conventional pi-section models. For this reason, the evaluated interactions are only representative for the frequency range up to the model is accurate and can lead to a misleading estimation of the dynamics [16].

At the same time, the increasing installation of converters coincides with the phase-out of conventional generation. This could give rise to an even higher influence of the mentioned converter dynamics in the power system dynamics and a more urgent need to consider the impact of the converter control loops on the overall stability. Otherwise, the fact of neglecting this influence in developing the network equivalents leads to the underestimation of the main phenomena and source of the 
problem, i.e., the control interaction.

Two problems arise. On the one hand, the limited scope of the harmonic impedance representation in commercial software arises concerns related to their possible inaccuracy and the resulting impact on system studies, such as harmonic or electromagnetic transient analysis. On the other hand, the limited range of model representation in the methodologies for stability study presented in literature arises uncertainty about the respective limitation in the estimated dynamics. And, as a consequence, the following questions appear: Does control dynamics influence in the overall network harmonic response? Which is the frequency range in which interactions might occur? Are existing tools and methods capturing the phenomena? Which is the consequence of the possible limitations in the tools? And, if needed, how can the interactions be studied with sufficient accuracy?

This paper analyses control interactions in meshed networks with multiple converters. The objective of the analysis is to evaluate the influence of converter control dynamics in the network resonances, and to study the limitations of current tools for the harmonic impedance. To do this, a systematic method is presented able to represent wide-band component dynamics and to capture converter control dynamics.

\section{INTEROPERABILITY STUDIES}

In the past, a lot of work has been done to obtain a highly accurate representation of the frequency-dependent behaviour of grid components for time-domain analysis [17]. The solution builds the state-space representation by obtaining the rational functions of each of the branches considering the different inputs and outputs. For interoperability studies, several converters models should be included in the same model and the time-domain solution would only indicate whether the system is stable or not and does not give relevant information for the system stability. Besides, there might be some confidentiality issues due to the implementation of different converter models, which might belong to different manufacturers, and it could require a long simulation time.

One solution to decrease the simulation time and to encompass other converters dynamics within an wider model would be to represent the surrounding network by a Thévenin equivalent, which would capture system dynamics within the range of interest. This frequency-dependent network is also requested by the manufacturer for different integration studies. Commercial power system analysis software typically includes a feature to obtain the harmonic impedance at a given connection point. The solution generates the system impedance for any electric network as seen from the interface point. In EMT tools, the software directly uses the RGLC data of the internal passive network components to obtain the harmonic impedance [18]. In any case, this solution only represents the dynamic behaviour from passive components in the network. Other tools represent the converter installation as a grey box by using an interface and thus, the converter is seen as a current source and the transformer is not considered as a passive component. This approach follows the IEC 61400-21 standard guideline of modelling converter-connected wind turbines as an harmonic current source [19].

However, for stability studies, it would be desirable to have a dynamic representation which could allow to assess the system stability in a more direct manner. In this respect, recent literature has focused on studying the dynamic interaction between converters and the implications on the system stability. To do so, system dynamics have been generally represented using two different approaches: state-space [12] or frequencydomain analysis [15]. Frequency-domain studies have some advantages because of the possibility to represent the different components as black-box models without information related to internal dynamics and parameters and entails several practical advantages due to the confidentiality of converter dynamic models. In this approach, the interconnection is represented by a nodal-admittance matrix as a multi-input multi-output system, in which the matrix elements are transfer functions. However, up to date, this admittance matrix have only represented the lumped parameters of conventional pisection models of lines [15], [20], [21].

\section{FREQUENCY-DEPENDENT PARAMETER ADMITTANCE MATRIX}

To overcome the previous limitations, i.e. the incapability to represent converter dynamics in frequency-scanning tools and the inaccurate frequency-dependent modelling of the network in typical stability studies, the paper presents a systematic methodology to represent network wide-band dynamics and to include converter control dynamics.

To do so, a formulation for the elements of the nodaladmittance matrix $\mathbf{Y}$ is presented. The formulation is based on the representation of interconnection elements by the well-known two-port-network representation. In this approach, admittance matrix elements are frequency-dependent variables. Interconnection elements, such as lines or transformers, are represented by the two-port $\mathrm{ABCD}$ parameters in (1); while elements connected to a given bus -loads, generators or converters- are represented by an equivalent frequencydependent admittance.

$$
\left(\begin{array}{l}
V_{2} \\
I_{2}
\end{array}\right)=\left(\begin{array}{ll}
A & B \\
C & D
\end{array}\right)\left(\begin{array}{l}
V_{1} \\
I_{1}
\end{array}\right)
$$

The FD matrix elements are calculated as,

$$
Y_{i j}= \begin{cases}y(\omega)_{i}+\sum \frac{1}{B(\omega)_{i k}}+\frac{A(\omega)_{i k}-1}{B(\omega)_{i k}} & \text { if } i=j \\ \frac{1}{B(\omega)_{i j}} & \text { if } i \neq j\end{cases}
$$

where $A(\omega)_{i j}$ and $B(\omega)_{i j}$ are the two-port elements and $y(\omega)_{i}$ are the components connected to a bus.

The use of ABCD parameters in the admittance matrix gives a more generic representation than previous formulation based on parallel and series connection of lumped parameters. As an example, the matrix formulation would remain identical independently the transmission line is modelled as a pi section, as a distributed-parameter line or the frequency-dependent model. Besides, the different nodes of the system are accessible so 


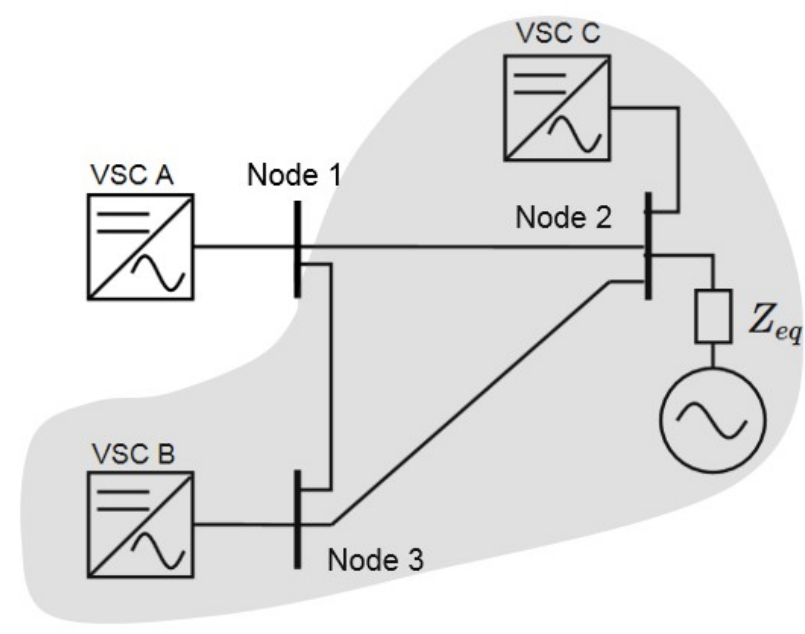

Fig. 1. Dynamic scheme of the VSC installation including control and plant dynamics.

that other components, modelled as a frequency-dependent responses, can be connected in a straightforward manner.

Once the FD admittance matrix is developed, the Thévenin equivalent ${ }^{1}$ is obtained by inverting the matrix and choosing the diagonal element at the bus of interest as,

$$
[\mathbf{Z}]=[\mathbf{Y}]^{-1}
$$

\section{STUDY CASE}

The system used as an example in this paper is a threenode meshed AC network. This network is composed of transmission lines, voltage source converters and a shortcircuit impedance ${ }^{2}$. The network is shown in Fig. 1.

As a detailed example, the presented methodology is implemented to develop the Thévenin equivalent of the network in the shaded area in Fig. 1. The nodal-admittance matrix of this system is,

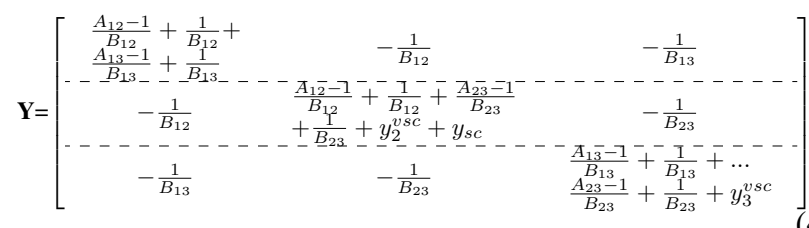

where $Y_{i}^{v s c}$ is the equivalent admittance from the given converter and $Y_{s c}$ is a short-circuit admittance $1 /(L s+R)$.

\section{A. Component modelling}

Models of the different components need to be accurately represented for a wide band of frequencies. It is not straightforward to determine the frequency range of such interactions because it depends on the influence of different components in the system damping. However, the higher damping at higher

\footnotetext{
${ }^{1}$ In the paper, Thévenin equivalents are analogous to the frequencydependent network equivalent or harmonic impedance.

${ }^{2}$ In the paper, the short-circuit impedance is only used in order to define the network as inductive and eliminate the capacitive dominance at the fundamental frequency due to the transmission lines.
}

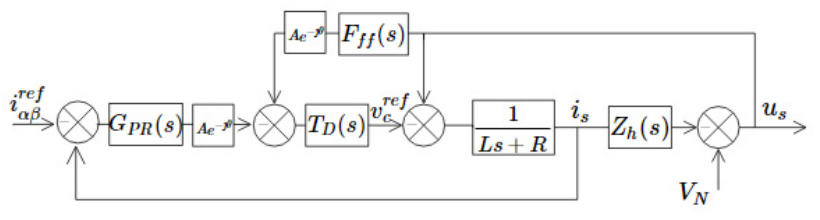

Fig. 2. Dynamic scheme of the VSC installation including control and plant dynamics.

frequencies suggests that interactions above $5-6 \mathrm{kHz}$ are very unlikely to occur due to the inherent damping and, thus, dynamics can be generally neglected.

1) Transmission lines: Transmission lines are represented by the distribution of frequency-dependent RGLC parameters in the phase domain. To do so, RGLC frequency-dependent parameters are calculated from the geometry and characteristics of the line. $A B C D$ parameters are then calculated to represent the distribution of parameters as,

$$
\begin{aligned}
& A=\cosh \gamma \\
& B=Z_{c} \sinh \gamma \\
& C=\frac{\sinh \gamma}{Z_{c}} \\
& D=A
\end{aligned}
$$

where

$$
\begin{array}{ll}
\gamma=\sqrt{z y} & \text { propagation constant } \\
Z_{c}=\sqrt{\frac{z}{y}} & \text { characteristic impedance } \\
z=R(\omega)+\jmath \omega L(\omega) & \text { series impedance } \\
y=\jmath \omega C(\omega) & \text { shunt admittance }
\end{array}
$$

The reader is referred to [22] for a detailed reference in line modelling and parameter calculation.

2) Voltage source converter: The connection of the converter to the network is represented by a current source with an equivalent admittance in parallel [23], [24]. The VSC admittance $Y_{v s c}$ captures control and circuit dynamics with a transfer function from the input AC voltage as the disturbance and the current through the reactor as the output. The considered control loops are the current controller $G_{c c}$, a filter in the voltage feed-forward to reject the influence of voltage harmonics $F_{f f}$ and a time delay representing inherent control delays due to modulation, calculation and discretization lags $T_{D}$ as sketched in Fig. 2. The VSC is designed to control each of the $a b c$ phases independently in the stationary frame. The scheme corresponds to one of the phases.

The transfer functions in Fig. 2 are defined below,

- The phase reactor is modelled by the equivalent phase impedance including the arm and leakage inductance $L=$ $\frac{L_{a r m}}{2}+L_{t r}$. Total values are $L_{a r m}=0.05$ and $L_{t r}=$ $0.0917 \mathrm{H}$ and $R=0.5 \Omega$.

- Proportional-resonant controller. The controller is tuned with $K_{P}=\alpha_{c} L$ and $K_{I}=\alpha_{i} K_{P}$, with $\alpha_{c}=800$ and $\alpha_{i}=100$.

$$
G_{P R}(s)=K_{P}+\frac{K_{I} s}{s^{2}+\omega_{N}^{2}}
$$


- Time Delay with $t_{d}=150 \mu s$.

$$
T_{D}(s)=\frac{-\frac{t_{d}}{2} s+1}{\frac{t_{d}}{2} s+1}
$$

- Feed-forward filter. The cut-off frequency $\omega_{c}$ is $2 \pi 350$ $\mathrm{rad} / \mathrm{s}$.

$$
F_{f f}(s)=\frac{\omega_{c}}{s+\omega_{c}}
$$

The equivalent admittance transfer function from the PCC voltage input to the PCC current output is hence calculated as,

$$
\begin{gathered}
Y_{v s c}(s)=\frac{i_{s}}{u_{s}}=\frac{1-T_{D}(s) F_{f f}(s)}{L s+R+T_{D}(s) G_{P R}(s)} \\
\text { V. RESULTS }
\end{gathered}
$$

The main objectives of this section are:

- to validate the presented methodology in developing the Thévenin equivalent of the network analytically,

- to demonstrate the need of representing converter control dynamics in the Thévenin equivalent,

- and to point out the limitations of widely used methodologies based on frequency scans from software.

To do so, the harmonic impedance of the network as seen from the node 1 in Fig. 1 is determined from two different power system analysis software and from the presented methodology. The resulting Thévenin equivalent represents the frequencydependent dynamics of the network inside the shaded area in Fig. 1.

\section{A. Network without converters}

First, before evaluating the influence of converters in the harmonic impedance as seen from node 1, the methodology is tested for the network without any converter connected, i.e., completely passive. The resulting harmonic impedance obtained from the presented methodology is compared with the frequency sweep obtained in the EMT software and results in Fig. 3 show a good correspondence between both harmonic impedances.

\section{B. Network with converters}

Grid resonances vary depending on how the different components are modelled and thus, different methods might give different results. Three harmonic impedances are built:

- (i) the Thévenin impedance obtained from the analytical methodology,

- (ii) the harmonic impedance obtained from the frequency scan in an EMT software with all the passive components and,

- (iii) the harmonic impedance obtained from the frequency sweep considering converters as current sources according to IEC $61400-21^{3}$.

Some concepts about the property of passivity in dynamic systems [25] are here recalled for a better understanding. In short, a linear system is passive if it dissipates energy and

\footnotetext{
${ }^{3}$ In the paper, PSCAD is used as the EMT software and DigSilent as the software the harmonic analysis following the IEC 61400-21 guidelines.
}

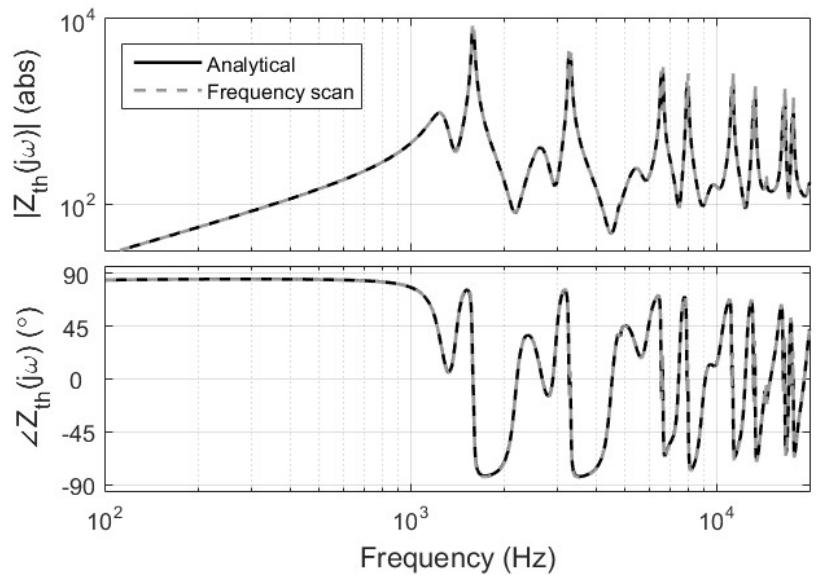

Fig. 3. Thévenin impedance of the network as seen from node 1: from the presented methodology (in solid black) and from the frequency scan in a EMT software (in dashed grey).

oscillations are attenuated. In electrical circuits, passivity is exhibited when their frequency-domain representation presents a phase angle within $-90^{\circ} \leq \varphi \leq 90^{\circ}$. Particularly, the system becomes more dissipative the further located to $\pm 90^{\circ}$ within the range and becomes active out of this boundary, i.e., might cause resonances to become unstable. When converters are connected to a network, the resulting frequency-dependent harmonic impedance varies depending on the influence of the control at a given frequency range.

Fig. 4 shows the three frequency-dependent Thévenin impedances. Comparing (iii) with (i)-(ii), the difference observed in the resonance frequencies corresponds to the fact that (iii) uses an interface for the complete model of the VSC at the point of connection, whereas (i) and (ii) represent the dynamics from the converter reactor. Hence, the VSC installation is considered in (iii) as a current source at the point of connection and the reactor is completely neglected [26]. This leads to a wrong estimation of grid resonances.

Regarding the (i) and (ii) frequency-dependent responses, there is a difference especially observable from the phase angle, which gives an indication of the system damping. This difference is due to the influence from the converters control. In the range between $100-1000 \mathrm{~Hz}$, (i) presents a higher damping, i.e. further to $90^{\circ}$ within the passive region, than (ii). This response is due to the fact that the current controller adds damping within its bandwidth. Above $1 \mathrm{kHz}$ and up to $4 \mathrm{kHz}$ approximately, the harmonic impedance from (i) shows a lower damping, i.e. closer to $-90^{\circ}$, than (ii). This decrease of damping is due to the action of control and system delays [27].

\section{Time-domain validation}

The main objective of a Thévenin equivalent is to reduce the network complexity at a node in such a way that dynamics are identically represented in the frequency range of interest. Therefore, the used network equivalent must reproduce the same dynamics of interest appearing in the original network. 


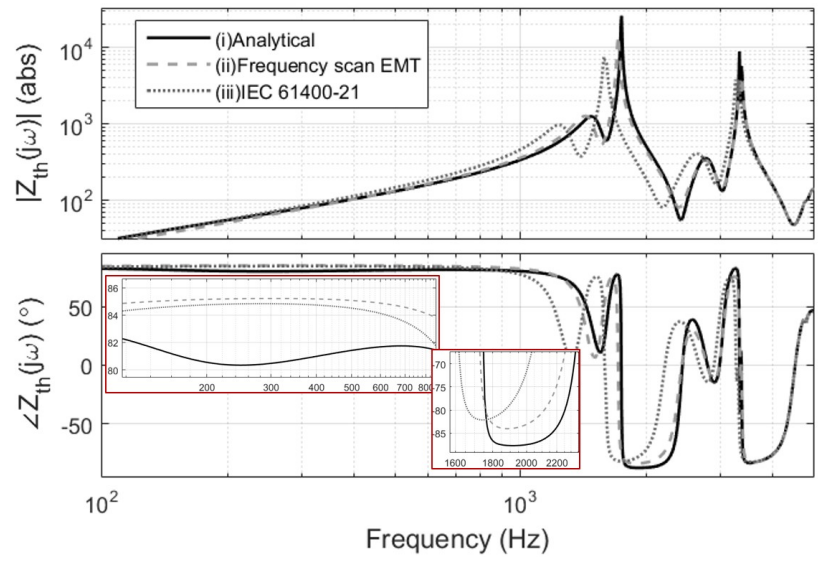

Fig. 4. Thévenin impedance of the network as seen from node 1: (i) from the presented methodology (in solid black) and (ii) from the frequency scan in a EMT software (in dashed grey) and (iii) from the frequency scan following IEC 61400-21 (in dotted grey).



Fig. 5. Time-domain simulation of the complete system and detailed capture during dynamics. At $\mathrm{t}=3 \mathrm{~s}$, there is a change in the topology. Detail of the oscillatory mode on the right.

The time-domain response of the original system, as shown in Fig. 1, is plotted in Fig. 5. The response shows an instability starting at $\mathrm{t}=3 \mathrm{~s}$, when the converter is connected to the network, with a frequency of $1880 \mathrm{~Hz}$ approximately.

Now, the system stability is analysed by using the obtained Thévenin impedances. To do this, the open-loop transfer function $Y_{1}^{v s c} Z_{t h, 1}$ is plotted. In this case, $Y_{v s c}$ corresponds to the converter connected at node 1 since we defined the interaction study at that node, and $Z_{t h}$ corresponds to the obtained Thévenin impedances from (i) and (ii). The two Bode curves are plotted in Fig. 6. The oscillatory behaviour can be estimated by looking at the gain crossover points, defined as the frequencies in which the amplitude crosses $0 \mathrm{~dB}$, and their respective phase margin or distance to $-180^{\circ}$. The curve using the Thévenin equivalent from (i), with control dynamics included, indicates an instability due to the negative phase margin at $1864 \mathrm{~Hz}$. On the other hand, the open-loop response with the harmonic impedance (ii) determines a low-damped oscillation at $1833 \mathrm{~Hz}$ due to the low positive phase margin.

At first glance, it is clear that using the harmonic impedance (ii) obtained from an EMT frequency scanning does not reveal the unstable behaviour of the interconnected system. Besides, the dynamic assessment using the harmonic impedance (i) identifies the instability and its frequency with a relative error in frequency around $1 \%$. From these observations, it

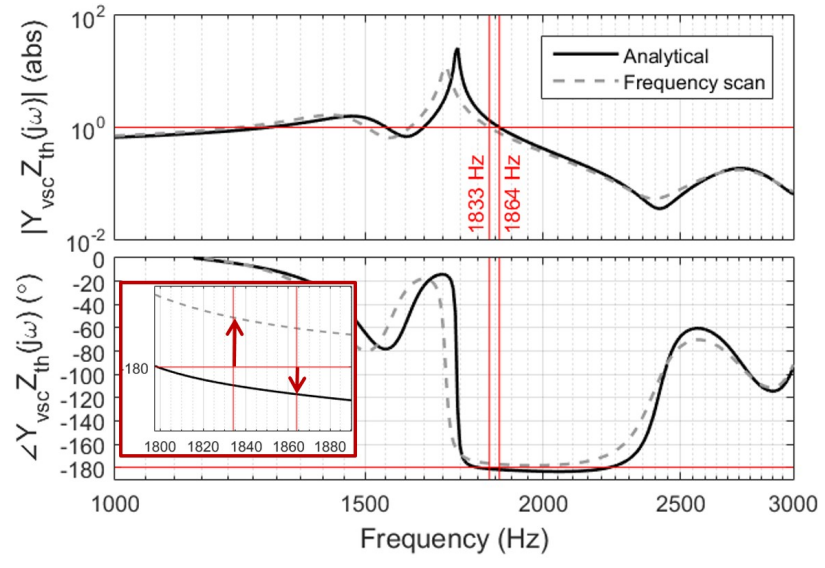

Fig. 6. Open-loop transfer functions of $Y Z$ for $Z$ as the Thévenin impedance obtained analytically from the proposed method (in black solid) and from the frequency scan in an EMT software (in dashed grey).

is demonstrated that control dynamics of parallel-connected converters need to be included in order to represent the dynamic behaviour of the system at frequencies above the fundamental.

Finally, the objective is to validate the accuracy of the developed Thévenin equivalent in representing the dynamics of the interconnected system. To do so, the time-domain response of the complete network as represented in Fig. 1 is compared with the time-domain response of the system with (ii) the Thévenin equivalent from the frequency scanning in the EMT software in Fig. 7 and (i) the analytically obtained Thévenin equivalent, in which control dynamics are included, in Fig. 8. These responses are obtained from the dynamic model sketched in Fig. 2, in which $Z_{h}$ is the Thévenin impedance, implemented in the software Matlab-Simulink.

Fig. 7 demonstrates the limitations of neglecting control dynamics. Despite the frequency scan being obtained from the same network in the EMT software, the time-domain response from the converter connected to the Thévenin (ii) differs from the response of the complete network in the damping and frequency of the oscillatory mode. Thus, the network reduction does not capture the unstable response. The damped oscillation corresponds to the oscillatory mode detected in Fig. 6 at 1833 $\mathrm{Hz}$.

Fig. 8 plots the time-domain response of the complete network in the EMT software and the converter connected to the analytical Thévenin equivalent (i). In this case, both responses are closer in comparison to the previous case. The use of the calculated harmonic impedance represents the instability and, therefore, the control interaction between the converters and the grid. The relative error between the estimated and resulting frequency of instability, 1864 and 1875 $\mathrm{Hz}$ respectively, is equal to $0.6 \%$. Besides, the amplification also differs.

The error in magnitude of the harmonic component corresponds to the fact that, first, the way the network is connected differs in both systems -in EMT the network is connected by 


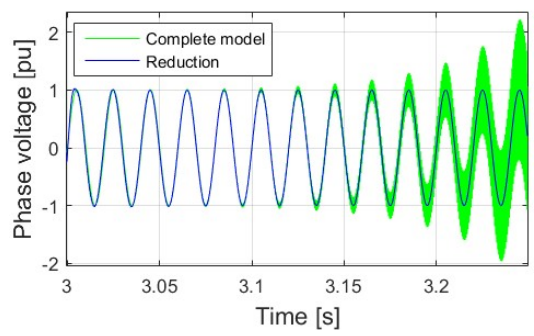

(a)

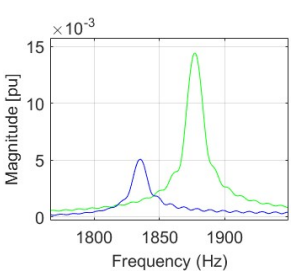

(b)
Fig. 7. Time-domain response of the phase voltage at the node 1 from the complete system simulated in an EMT software (in lighter green) and the response of the dynamic system with the harmonic impedance measured from the EMT frequency scan tool (in darker blue), and fft applied to the both responses in the window $t=3 \div 3.1 \mathrm{~s}$ in (b).

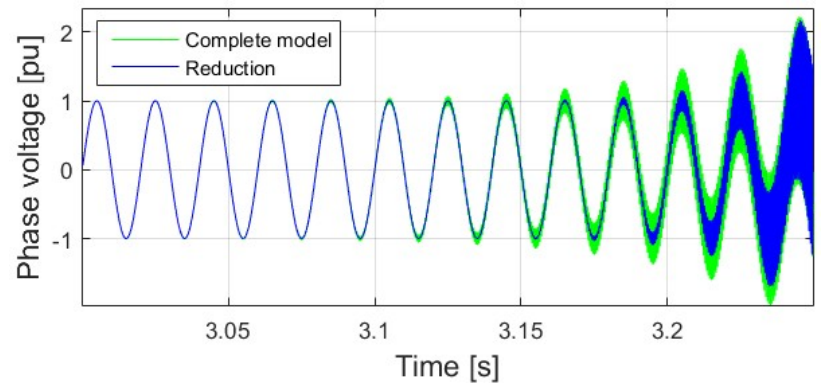

(a)

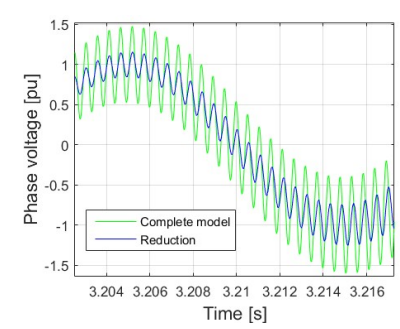

(b)

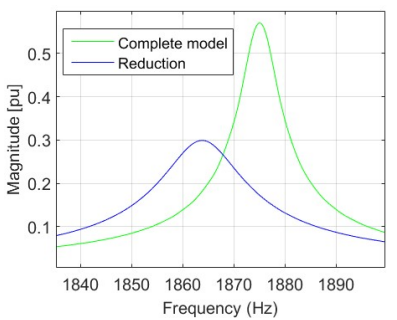

(c)
Fig. 8. Time-domain response of the phase voltage at the node 1 from the complete system simulated in an EMT software (in lighter green) and the response of the dynamic system with the harmonic impedance obtained analytically (in darker blue) in (a), detailed window of the time-domain response in (b), and fft applied to the both responses in the window $t=3 \div 3.25 \mathrm{~s}$ in (c).

breakers and in the dynamic model the transfer function $Z_{t h}$ is connected- and, second, the oscillation is also propagated to the other converters and fed through their control. Thus, the component appears at their output as result of the feedforward of background harmonics. The network reduction only represents network resonances and does not capture the voltage synthesization of the parallel converters. Besides, other differences such as the use of different solvers, the effect of the integration step, the dynamic implementation of some blocks -e.g. delays-, or the use of curve fitting and the passivity enforcement for the time-domain solution might influence in the result.

\section{CONCLUSIONS}

In industry, interoperability studies in networks with multiple converters raises several concerns due to modelling and simulation requirements and protection of the dynamic models. Up to date, the interaction between a converter and a given network is studied by means of representing the network by a frequency-dependent network equivalent. However, this paper demonstrates that the current methodologies and guidelines would not reveal the resulting interactions in systems with multiple converters because the frequency scan neglects control dynamics.

In the paper, we present a generic and systematic analytical methodology to analyse such interactions in the frequencydomain while ensuring a good representation of wide-band dynamics and including converter control dynamics. Results not only show the estimation of the dynamic behaviour seen in an EMT simulation but also demonstrates that the analytically developed frequency-dependent equivalent captures the same phenomena and time-domain response than the complete network built in an EMT tool. This makes the presented methodology attractive to analyse the system stability and to obtain network equivalents which includes control dynamics from parallel converters while protecting their data.

\section{REFERENCES}

[1] S. Menth and M. Meyer, "Low-frequency power oscillations in electric railway systems," Elektrische Bahnen, vol. 104, no. 5, pp. 216-221, 2006.

[2] E. Mollerstedt and B. Bernhardsson, "Out of control because of harmonics-an analysis of the harmonic response of an inverter locomotive," IEEE Control Systems, vol. 20, no. 4, pp. 70-81, August 2000.

[3] H. Enslin and P. J. Heskes, "Harmonic interaction between a large number of distributed power inverters and the distribution network," IEEE Transactions on Power Electronics, vol. 19, no. 6, pp. 1586-1593, November 2004.

[4] R. Teodorescu, M. Liserre, and P. Rodríguez, Grid Converters for Photovoltaic and Wind Power Systems, J. W. . Sons, Ed., 2011.

[5] C. Buchhagen, C. Rauscher, A. Menze, and J. Jung, "BorWin1 - First Experiences with harmonics interactions in converter dominated grids," in International ETG Congress 2015 in Bonn., November 2015, 7 pages.

[6] H. Saad and (RTE). (2016, August) Performance analysis of inelfe link with control replicas [accessed on 26th september 2016]. $</ /$ sites.google.com/site/hvdcreplicaworkshop/workshop-presentations $>$.

[7] ENTSO-E. Draft Network Code on HVDC Connections and DCconnected Power Park Modules (Article 27).

[8] G. Alvarez-Cordero, A. Bachiller, A. Gómez-Expósito, J. RosendoMacías, and Gómez-Simón, "A methodology for harmonic impedance in large power systems. application to the filters of a vsc." Proceedings of Cigré Sessions 2012 in Paris, 2012, 7 pages.

[9] L. H. Kocewiak, J. Hjerrild, and C. L. Bak, "Wind turbine converter control interaction with complex wind farm systems," IET Renewable Power Generation, vol. 7, no. 4, pp. 380-389, July 2013.

[10] J. Glasdam and L. H. Kocewiak, "Control System interaction in the VSC-HVDC Grid Connected Offshore Wind Power Plant," in Cigre B4 Simposium. Across borders - HVDC Systems and Market Integration in Lund., May 2015, 8 pages.

[11] S. Chaudhury, F. F. et R. Teodorescu, J. Guerrero, C. Bak, L. Kocewiak, and C. Jensen, "Harmonic Resonance in Wind Power Plants: Modeling, Analysis and Active Mitigation Methods," in Proceedings of the IEEE PowerTech Eindhoven 2015, 2015, 6 pages.

[12] L. Kunjumuhammed, C. Bikal, C.Oates, and K.J.Dyke, "Electrical Oscillations in Wind Farm Systems: Analysis and Insight Based on Detailed Modeling," IEEE Transactions on Sustainable Energy, vol. 7, no. 1, pp. 51-62, January 2016. 
[13] A. Bayo-Salas, J. Beerten, J. Rimez, and D. V. Hertem, "Analysis of control interactions in multi-infeed VSC HVDC connections," IET Generation, Transmission \& Distribution, vol. 10, no. 6, pp. 1336-1344, 2016.

[14] C. Wan, M. Huang, C. Tse, and X. Ruan, "Effects of Interaction of Power Converters Coupled via Power Grid: A Design-Oriented Study," IEEE Transanctions on Power Electronics, vol. 30, no. 7, pp. 3589-3600, July 2015.

[15] X. Wang, F. Blaabjerg, and W. Wu, "Modeling and analysis of harmonic stability in an AC power-electronics-based power system," IEEE Transactions on Power Electronics, vol. 29, no. 12, pp. 6421-6432, December 2014.

[16] J. Beerten, S. D’Arco, and J. Suul, "Frequency-Dependent Cable Modelling for Small-Signal Stability Analysis of VSC-HVDC Systems," IET Generation Transmission \& Distribution, vol. 10, no. 6, pp. 1370-1381, 2016.

[17] S. Grivet-Tralocia and B. Gustavsen, Passive Macromodeling: Theory and Applications, Wiley, Ed., 2016.

[18] PSCAD, "Pscad user's guide v4.6.0," Tech. Rep., 2013.

[19] "Iec 61400-21 wind turbines - part 21: Measurement and assessment of power quality characteristics of grid connected wind turbines," IEC, Tech. Rep., 2008.

[20] W. Xu, Z. Huang, Y. Cui, and H. Wang, "Harmonic Resonance Mode Analysis," IEEE Transactions on Power Delivery, vol. 20, no. 2, pp. 1182-1190, April 2005.

[21] A. Pana and A. Baloi, "Analysis Resonant Frequencies in AC Distribution Networks - A numerical example Part I- Harmonic Nodal Admittance Matrix Method," in Proceedings of 6th World Conference on educational Scienses, Elsevier, Ed., vol. 191, June 2015, pp. 1225-1232.

[22] J. Martinez-Velasco, Power system transients: Parameter determination, C. Press, Ed. Taylor \& Francis Group, 2010.

[23] L. Harnefors, M. Bongiorno, and S. Lundberg, "Input-admittance calculation and shaping for controlled voltage-source converters," IEEE Transactions on Industrial Electronics, vol. 54, no. 6, pp. 3323-3334, December 2007.

[24] J. Sun, "Impedance-based stability criterion for grid-connected inverters," IEEE Transactions on Power Electronics, vol. 26, no. 11, pp. 30753078, November 2011.

[25] J. C. Willems, "Dissipative dynamical systems, part i: General theory," Archive for Rational Mechanics and Analysis, vol. 45, no. 5, pp. 321351, January 1972.

[26] L. Kocewiak, "Harmonics in large offshore wind farms," Ph.D. dissertation, 2012.

[27] L. Harnefors, X. Wang, A. Yepes, and F. Blaabjerg, "Passivity-based stability assessment of grid-connected vscs - an overview," IEEE Journal of emerging and selected topics in power electronics, vol. 4, no. 1, pp. 116-125, 2016. 